\title{
Photodynamic therapy of early stage oral cavity and oropharynx neoplasms: an outcome analysis of 170 patients
}

\author{
Baris Karakullukcu $\cdot$ Kim van Oudenaarde $\cdot$ \\ Marcel P. Copper · W. M. C. Klop · Robert van Veen • \\ Maarten Wildeman $\cdot$ I. Bing Tan
}

Received: 13 April 2010 / Accepted: 2 August 2010 / Published online: 13 August 2010

(C) The Author(s) 2010. This article is published with open access at Springerlink.com

\begin{abstract}
The indications of photodynamic therapy (PDT) of oral cavity and oropharynx neoplasms are not well defined. The main reason is that the success rates are not well established. The current paper analyzes our institutional experience of early stage oral cavity and oropharynx neoplasms (Tis-T2) to identify the success rates for each subgroup according to $\mathrm{T}$ stage, primary or non-primary treatment and subsites. In total, 170 patients with 226 lesions are treated with PDT. From these lesions, 95 are primary neoplasms, 131 were non-primaries (recurrences and multiple primaries). The overall response rate is $90.7 \%$ with a complete response rate of $70.8 \%$. Subgroup analysis identified oral tongue, floor of mouth sites with more favorable outcome. PDT has more favorable results with certain subsites and with previously untreated lesions. However, PDT can find its place for treating lesions in previously treated areas with acceptable results.
\end{abstract}

B. Karakullukcu · W. M. C. Klop · M. Wildeman · I. Bing Tan Department of Head and Neck Oncology and Surgery,

The Netherlands Cancer Institute, Antoni van Leeuwenhoek Hospital, Amsterdam, The Netherlands

K. van Oudenaarde

Amsterdam Medical Centre,

University of Amsterdam, Amsterdam, The Netherlands

M. P. Copper

Sint Antonius Hospital, Nieuwegein, The Netherlands

R. van Veen

Centre for Optical Diagnosis and Treatment,

Erasmus University, Rotterdam, The Netherlands

B. Karakullukcu ( $\square)$

Afdeling Hoofd Hals Chirurgie, The Netherlands Cancer Institute, Plesmanlaan 121, 1066 CX Amsterdam, The Netherlands

e-mail: b.karakullukcu@nki.nl
Keywords Oral cancer - Oropharynx cancer .

Photodynamic therapy $\cdot$ mTHPC $\cdot$ Early stage neoplasm

\section{Introduction}

Photodynamic therapy (PDT) is a relatively new method for management of head and neck neoplasms. Surgery and radiotherapy are long accepted as the standard treatment for head and neck neoplasms with long successful track records $[1,2]$. This standard approach has an established success rate approaching $95 \%$ for early stage neoplasms [3, 4]. The upper aerodigestive tract has important functions, such as respiration, swallowing and phonation. Standard treatment of the neoplasms of head and neck region, although not frequent with superficial neoplasms, might compromise one or more of these functions. Especially with repeated treatments for recurrent and multiple primaries, these problems become more evident [5-8]. New reconstructive techniques, less invasive surgical modalities such as laser or robot-assisted endoscopic surgery, more precise delivery of radiation with techniques, such as intensity-modified radiation treatment (IMRT) all aim to decrease the morbidity rate [9-12].

PDT is searching its role in the era of conservation surgery. The advantage of PDT is local treatment without long-term systemic effects. The photosensitizing drug is activated by the light delivered directly onto the neoplasm sparing the surrounding normal mucosa. Protection of the surrounding tissue is further assured by shielding with wet sponges or special shielding waxes.

An additional advantage of PDT is that it shows its effect via cytotoxicity rather than destructive effects. This means that when cancer cells are eliminated via apoptosis the extracellular matrix remains forming a scaffold for the 
surrounding mucosal tissue to advance over [13, 14]. Scar formation is minimal and the native tissue that replaces the cancer cells maintains its normal functions limiting the functional loss significantly.

Perhaps, the most important aspect of PDT is its repeatability. PDT can be applied to the same area without accumulative destructive effects [15-17]. It also does not negatively effect further treatments, such as radiation or chemotherapy [15], leaving further treatment options open.

Despite these advantages PDT remains unknown to many head and neck oncologists [17-20]. Because PDT is searching its place in the management cascade of head and neck oncology, we believe it is important to have a basic knowledge of what degree of success can be expected in various clinical scenarios. The current paper is aiming to analyze retrospectively a subgroup of patients with early stage oral cavity and oropharynx neoplasms to demonstrate what percentage of success to expect with PDT. This analysis includes neoplasms of different subsites of the oral cavity and oropharynx, primary neoplasms versus recurrences or multiple primary neoplasms in previously conventionally treated fields.

\section{Materials and methods}

The registry of patients (294 patients between 1996 and 2008) treated with PDT was retrospectively screened to identify patients with early stage (Tis, T1, T2) oral cavity and oropharynx squamous cell cancers or carcinoma in situ. One hundred and seventy patients were included in the analysis.

All patients were subject to pretreatment evaluation according to the guidelines of our institute.

Pre-treatment work-up

Standard minimum evaluation consisted of biopsy to determine the histological diagnosis of squamous cell cancer or carcinoma in situ (Tis); X-ray of the thorax; ultrasound (US) of the neck and fine-needle aspiration (FNA) to determine nodal metastasis situation. Tumor thickness is measured either by US or magnetic resonance imaging (MRI). The whole surface of the oral cavity tumors are screened with an oral US probe to determine the deepest point of infiltration. Only tumors that have less than or equal to $5 \mathrm{~mm}$ depth of invasion were treated with surface PDT. Oropharyngeal tumors could not be reached by US and, therefore, evaluated by the less accurate method of MRI. If the tumors cannot be visualized on the MR images or there is a surface of contrast uptake, but no evidence of deep invasion, the patients received PDT. The cut-off depth of
$5 \mathrm{~mm}$ is decided to enable treatment of $5 \mathrm{~mm}$ margin deeper than the tumor. The total $10 \mathrm{~mm}$ is the average depth of penetration of the $652 \mathrm{~nm}$ light used to activate the photosensitizer.

The work-up for regional lymphatic status is US of the neck and FNA of suspected lymph nodes. All patients who received PDT had N0 stage according to US criteria. The guidelines of our institute involve no prophylactic neck dissections for T1 and T2 oral cavity tumors if they are candidates for transoral excisions or PDT. The neck node status is followed by US combined with FNA at 6-month intervals. Patients with suspected lymph nodes receive modified radical neck dissection.

Patients were informed of potential benefits and risks of the treatment and instructed about light avoidance measures and supplied with a light meter to measure ambient light.mTHPC (temoporfin, Foscan ${ }^{\circledR}$, Biolytec Pharma Ltd., Dublin, Ireland) at a dose of $0.15 \mathrm{mg} / \mathrm{kg}$ was injected to a deep vein with slow infusion. Patients were discharged home after the injection. Illumination took place $96 \mathrm{~h}$ after the mTHPC injection. Light is delivered by a diode laser at $652 \mathrm{~nm}$. Dose delivered is $20 \mathrm{~J} / \mathrm{cm}^{2}$ with a fluence rate of $100 \mathrm{~mW} / \mathrm{cm}^{2}$. Preferred method is to deliver light with one spot delivered via a microlens. If one spot illumination is not applicable due to the shape or location of the neoplasm multiple spots were used. The lesion plus $5 \mathrm{~mm}$ margin of normal appearing mucosa is illuminated. All patients received corticosteroids and pain management. Hospital stay depended on the functional limitations after PDT due to location and extent of the neoplasm, as well as edema due to PDT.

Tumor response is analyzed according to the World Health Organization (WHO) criteria [20]:

Complete response (CR): the disappearance of all known disease

Partial response (PR): $50 \%$ or more decrease in the dimensions of the tumor

No response (NR): less than $50 \%$ decrease or less than $25 \%$ increase in tumor dimensions

Progressive disease (PD): $25 \%$ or more increase in the size of one or more measurable lesions or the appearance of new lesions

All responses have to be confirmed by two observations not $<4$ weeks apart. Overall response (OR) is the sum of complete and partial responders.

\section{Follow-up}

There is a follow-up schedule of 1, 2, 4, 8, 16, 24, 36, 52 weeks after PDT, followed by routine controls every 4 months for a total of 5 years. 


\section{Results}

Patients

Of the 170 patients evaluated, 80 were female and 90 were male. The average age at treatment was 60.5 years. A total of 226 neoplasms was treated with PDT, 35 patients are treated for 2 or more neoplasms. Of the evaluated neoplasms, 95 were primary neoplasms, 131 were non-primary neoplasms consisting of 65 recurrences, $46 \mathrm{~s}, 9$ third and 3 fourth primary neoplasms and of 8 residual neoplasms after initial treatment. Previous treatments within the non-primary neoplasm group $(n=131)$ include: radiotherapy $48.1 \%$, chemoradiation $22.7 \%$, surgery $75.6 \%$ and previous PDT $30.6 \%$.

\section{Tumor response}

The OR rate for all neoplasms is $90.7 \%$ (95\% CI $86.2-$ $94.2 \%$ ) with a CR rate of $70.8 \%$ (95\% CI 64.4-76.6\%). In Table 1, the responses are listed per site, per stage and primary or non-primary. The median local disease-free interval for all of the CR cases is 102.0 months (95\% CI 89.6-114.3 months). 2- and 5-year local disease-free survival rates are 74 and $61 \%$, respectively.

\section{Analysis per T classification}

Tis lesions have an OR rate of 94.5\% (95\% CI 86.6-98.5\%), with a CR rate of $79.5 \%$ (95\% CI $68.4-88.0 \%)$; T1 neoplasms have an OR rate of $90.9 \%$ (95\% CI 84.3-95.4\%) with a CR rate of $68.6 \%(95 \%$ CI $59.5-76.7 \%)$; and for the T2 neoplasms, the OR rate is $81.3 \%$ (95\% CI $63.6-92.8 \%$ ) with a CR of $59.4 \%$ (95\% CI 40.7-76.3\%). The variations in tumor response between the stages are only statistically significant for Tis versus T2 stage tumors $(P<0.05)$. The mean local disease-free interval is 65.7 months (95\% CI 49.3-82.2 months) for the Tis group, 109.1 months (95\% CI 93.1-125.0 months) for T1 and 113.4 months (95\% CI 81.9-144.9 months) for T2 (Fig. 1). The mean survival time is 92.2 months (95\% CI 79.3-105.0 months) for dys/CIS, 98.4 months (95\% CI 84.6-112.2 months) for $\mathrm{T} 1$ and 78.7 months (95\% CI 54.2-103.2 months) for T2 tumors.

\section{Sub-site analysis}

In Table 1, an overview of all treated sites is shown. The CR rate is highest for the oral tongue site [94.4\% (95\% CI $81.3-99.3 \%)]$, the floor of mouth [76.9\% (95\% CI 64.8$86.5 \%)]$ and the soft palate [75.9\% (95\% CI 56.5-89.7\%)] and lowest for the nasal cavity [37.5\% (95\% CI 0.9-75.5\%)], the alveolar process $[42.1 \%$ (95\% CI $20.3-66.5 \%)]$ and the

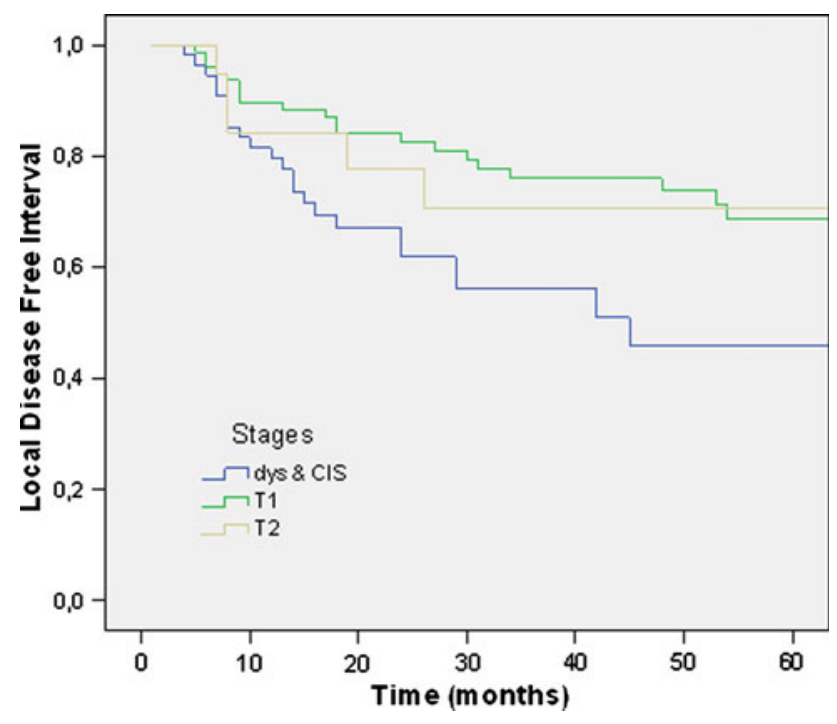

Fig. 1 Local disease-free interval per T stage of neoplasms

buccal mucosa [57.7\% (95\% CI 36.9-76.7\%)]. Oral tongue has significantly better response rate than the rest of the group $(P<0.05)$ and alveolar process has significantly lower response rate than the rest of the group $(P<0.05)$.

\section{Primary neoplasms versus non-primary neoplasms}

Primary neoplasms have an OR rate of $96.8 \%(95 \% \mathrm{CI}$ 91.1-99.3\%) with a CR rate of $77.9 \%$ (95\% CI $68.2-$ $85.8 \%$ ). For the non-primary neoplasms, the OR rate is $86.2 \%$ (95\% CI 79.2-91.7\%) and the CR rate is $65.6 \%$ (95\% CI 56.9-73.7\%).

Neoplasms located in an irradiated field $(n=64)$ have a CR rate of $57.8 \%$ (95\% CI 44.8-70.1\%); neoplasms located in an area that was treated before with surgery $(n=99)$ have a CR rate of $62.6 \%$ (95\% CI 52.3-72.2\%). There is no statistical difference.

The mean local disease-free interval for the primary neoplasms with a CR is 117.8 months (95\% CI 102.1133.6 months). For the non-primary neoplasms with a CR, the interval is 84.9 months (95\% CI 67.2-102.7 months). 1, 2 and 5-year survival of patients with primary neoplasms are 90,85 and $74 \%$, respectively. For non-primary neoplasms disease-free survival at 1, 2 and 5 years are 81, 64 and $48 \%$, respectively (Fig. 2). The difference in local disease-free survival for primary neoplasms versus non-primary neoplasms is statistically significant $(P<0.001)$.

The overall mean survival time for the primary neoplasms was 120.4 months (95\% CI 106.4-134.4 months) and for the non-primary neoplasms, this was 82.1 months (95\% CI 67.7-96.5 months) (Fig. 3). The difference in survival time for primary neoplasms versus non-primary neoplasms is statistically significant with $P<0.05$. 
Table 1 Overall and complete clinical responses to PDT per T stage and site

\begin{tabular}{|c|c|c|c|c|c|c|c|c|c|c|c|c|c|c|c|c|}
\hline \multirow[t]{4}{*}{ SITE } & \multicolumn{16}{|l|}{ Stage } \\
\hline & \multicolumn{4}{|c|}{ Dysplasia } & \multicolumn{4}{|l|}{$\mathrm{T} 1$} & \multicolumn{4}{|l|}{$\mathrm{T} 2$} & \multicolumn{4}{|c|}{ All stages } \\
\hline & \multicolumn{2}{|l|}{ OR } & \multicolumn{2}{|l|}{$\mathrm{CR}$} & \multicolumn{2}{|l|}{ OR } & \multicolumn{2}{|l|}{$\mathrm{CR}$} & \multicolumn{2}{|l|}{ OR } & \multicolumn{2}{|l|}{$\mathrm{CR}$} & \multicolumn{2}{|l|}{ OR } & \multicolumn{2}{|l|}{$\mathrm{CR}$} \\
\hline & $\mathrm{OR} / n$ & $\%$ & $\mathrm{CR} / n$ & $\%$ & $\mathrm{OR} / n$ & $\%$ & $\mathrm{CR} / n$ & $\%$ & $\mathrm{OR} / n$ & $\%$ & $\mathrm{CR} / n$ & $\%$ & $\mathrm{OR} / n$ & $\%$ & $\mathrm{CR} / n$ & $\%$ \\
\hline \multicolumn{17}{|c|}{ Floor of mouth $n=65$} \\
\hline Total & $24 / 25$ & 96.0 & $22 / 25$ & 88.0 & $29 / 30$ & 96.7 & $20 / 30$ & 66.7 & $9 / 10$ & 90.0 & $8 / 9$ & 80.0 & $62 / 65$ & 95.4 & $50 / 65$ & 76.9 \\
\hline Primary & $7 / 7$ & 100 & $6 / 7$ & 85.7 & $20 / 20$ & 100 & $13 / 20$ & 65.0 & $6 / 6$ & 100 & $5 / 6$ & 83.3 & $33 / 33$ & 100 & $24 / 33$ & 72.7 \\
\hline Non-primary & $17 / 18$ & 94.5 & $16 / 18$ & 88.9 & $9 / 10$ & 90.0 & $7 / 10$ & 70.0 & $3 / 4$ & 75.0 & $3 / 4$ & 75.0 & $29 / 32$ & 90.6 & $26 / 32$ & 81.3 \\
\hline \multicolumn{17}{|c|}{ Oral tongue $n=36$} \\
\hline Total & $12 / 12$ & 100 & $10 / 12$ & 83.3 & $24 / 24$ & 100 & $24 / 24$ & 100 & - & - & - & - & $36 / 36$ & 100 & $34 / 36$ & 94.4 \\
\hline Primary & $5 / 5$ & 100 & $4 / 5$ & 80.0 & $17 / 17$ & 100 & $17 / 17$ & 100 & - & - & - & - & $22 / 22$ & 100 & $21 / 22$ & 95.5 \\
\hline Non-primary & $7 / 7$ & 100 & $6 / 7$ & 85.7 & $7 / 7$ & 100 & $7 / 7$ & 100 & - & - & - & - & $14 / 14$ & 100 & $13 / 14$ & 92.9 \\
\hline \multicolumn{17}{|c|}{ Soft palate $n=29$} \\
\hline Total & $8 / 8$ & 100 & $8 / 8$ & 100 & $14 / 15$ & 93.3 & $10 / 15$ & 66.7 & $4 / 6$ & 66.7 & $4 / 6$ & 66.7 & $26 / 29$ & 89.7 & $22 / 29$ & 75.9 \\
\hline Primary & $2 / 2$ & 100 & $2 / 2$ & 100 & $7 / 7$ & 100 & $4 / 7$ & 57.1 & $2 / 3$ & 66.7 & $2 / 3$ & 66.7 & $11 / 12$ & 91.7 & $8 / 12$ & 66.7 \\
\hline Non-primary & $6 / 6$ & 100 & $6 / 6$ & 100 & $7 / 8$ & 87.5 & $6 / 8$ & 75.0 & $2 / 3$ & 66.7 & $2 / 3$ & 66.7 & $15 / 17$ & 88.2 & $14 / 17$ & 82.4 \\
\hline \multicolumn{17}{|c|}{ Buccal mucosa $n=26$} \\
\hline Total & $4 / 5$ & 80.0 & $3 / 5$ & 60.0 & $13 / 15$ & 86.7 & $10 / 15$ & 66.7 & $6 / 6$ & 100 & $2 / 6$ & 33.3 & $23 / 26$ & 88.5 & $15 / 26$ & 57.7 \\
\hline Primary & - & - & - & - & $7 / 8$ & 87.5 & $5 / 8$ & 62.5 & $3 / 3$ & $/ 100$ & $0 / 3$ & 0 & $10 / 11$ & 90.9 & $5 / 11$ & 45.5 \\
\hline Non-primary & $4 / 5$ & 80 & $3 / 5$ & 60.0 & $6 / 7$ & 85.7 & $5 / 7$ & 71.4 & $3 / 3$ & 100 & $2 / 3$ & 66.7 & $13 / 15$ & 86.7 & $10 / 15$ & 66.7 \\
\hline \multicolumn{17}{|c|}{ Oropharynx $n=26$} \\
\hline Total & $5 / 7$ & 71.4 & $4 / 7$ & 57.1 & $11 / 13$ & 84.6 & $9 / 13$ & 69.2 & $6 / 6$ & 100 & $4 / 6$ & 66.7 & $22 / 26$ & 84.6 & $17 / 26$ & 65.4 \\
\hline Primary & $3 / 3$ & 100 & $3 / 3$ & 100 & $4 / 4$ & 100 & $4 / 4$ & 100 & - & - & - & - & $7 / 7$ & 100 & $7 / 7$ & 100 \\
\hline Non-primary & $2 / 4$ & 50.0 & $1 / 4$ & 25.0 & $7 / 9$ & 77.8 & $5 / 9$ & 55.6 & $6 / 6$ & 100 & $4 / 6$ & 66.7 & 1,519 & 78.9 & $10 / 19$ & 52.6 \\
\hline Alveolar proces & $\mathrm{s} n=19$ & & & & & & & & & & & & & & & \\
\hline Total & $10 / 10$ & 100 & $6 / 10$ & 60.0 & $4 / 7$ & 57.1 & $2 / 7$ & 28.6 & $0 / 2$ & 0 & - & - & $14 / 19$ & 73.7 & $8 / 19$ & 42.1 \\
\hline Primary & $1 / 1$ & 100 & $1 / 1$ & 100 & $1 / 1$ & 100 & $1 / 1$ & 100 & $0 / 1$ & 0 & - & - & $2 / 3$ & 66.7 & $2 / 3$ & 66.7 \\
\hline Non-primary & $9 / 9$ & 100 & $5 / 9$ & 55.6 & $3 / 6$ & 50.0 & $1 / 6$ & 16.7 & $0 / 1$ & 0 & - & - & $12 / 16$ & 75.0 & $6 / 16$ & 37.5 \\
\hline Retromolar trigc & on $n=9$ & & & & & & & & & & & & & & & \\
\hline Total & $3 / 3$ & 100 & $3 / 3$ & 100 & $5 / 5$ & 100 & $2 / 5$ & 10.0 & $1 / 1$ & 100 & $1 / 1$ & 100 & $9 / 9$ & 100 & $6 / 9$ & 66.7 \\
\hline Primary & $2 / 2$ & 100 & $2 / 2$ & 100 & $2 / 2$ & 100 & $2 / 2$ & 100 & $1 / 1$ & 100 & $1 / 1$ & 100 & $5 / 5$ & 100 & $5 / 5$ & 100 \\
\hline Non-primary & $1 / 1$ & 100 & $1 / 1$ & 100 & $3 / 3$ & 100 & $0 / 3$ & 0 & - & - & - & - & $4 / 4$ & 100 & $1 / 4$ & 25.0 \\
\hline Hard palate $n=$ & & & & & & & & & & & & & & & & \\
\hline Total & $3 / 3$ & 100 & $2 / 3$ & 66.7 & $5 / 5$ & 100 & $3 / 5$ & 60.0 & - & - & - & - & $8 / 8$ & 100 & $5 / 8$ & 62.5 \\
\hline Primary & $1 / 1$ & 100 & $1 / 1$ & 100 & $1 / 1$ & 100 & $1 / 1$ & 100 & - & - & - & - & $2 / 2$ & 100 & $2 / 2$ & 100 \\
\hline Non-primary & $0 / 2$ & 0 & $0 / 2$ & 0 & $4 / 4$ & 100 & $2 / 4$ & 50.0 & - & - & - & - & $6 / 6$ & 100 & $3 / 6$ & 50.0 \\
\hline Nasal cavity $n=$ & & & & & & & & & & & & & & & & \\
\hline Total & - & - & - & - & $5 / 7$ & 71.4 & $3 / 7$ & 42.9 & $0 / 1$ & 0 & - & - & $5 / 8$ & 62.5 & $3 / 8$ & 37.5 \\
\hline Primary & - & - & - & - & - & - & - & - & - & - & - & - & - & - & - & - \\
\hline Non-primary & - & - & - & - & $5 / 7$ & 71.4 & $3 / 7$ & 42.9 & $0 / 1$ & 0 & - & - & $5 / 8$ & 62.5 & $3 / 8$ & 37.5 \\
\hline Subtotal & & & & & & & & & & & & & & & & \\
\hline Primary & $21 / 21$ & 100 & $19 / 21$ & 90.5 & $59 / 60$ & 98.3 & $47 / 60$ & 78.3 & $12 / 14$ & 85.7 & $8 / 14$ & 57.1 & $92 / 95$ & 96.8 & $74 / 95$ & 77.9 \\
\hline Non-primary & $48 / 52$ & 92.3 & $39 / 52$ & 75.0 & $51 / 61$ & 83.6 & $36 / 61$ & 59.0 & $14 / 18$ & 77.8 & $11 / 18$ & 61.1 & $113 / 131$ & 86.3 & $86 / 131$ & 65.6 \\
\hline Total & $69 / 73$ & 94.5 & $58 / 73$ & 79.5 & $110 / 121$ & 90.9 & $83 / 121$ & 68.6 & $26 / 32$ & 81.3 & $19 / 32$ & 59.4 & $205 / 226$ & 90.7 & $160 / 226$ & 70.8 \\
\hline
\end{tabular}

$O R$ overall response, $C R$ complete response, $O R / n$ number of overall response/total number of patients treated in this subgroup, $C R / n$ number of complete responders/total number of patients treated in this subgroup 


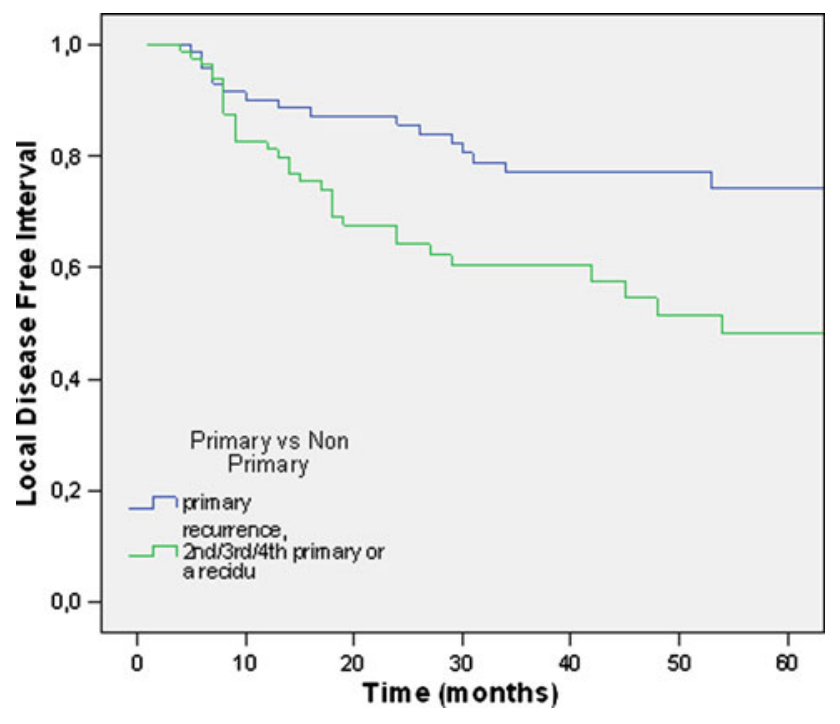

Fig. 2 Local disease-free interval, comparing primary neoplasms versus non-primary neoplasms

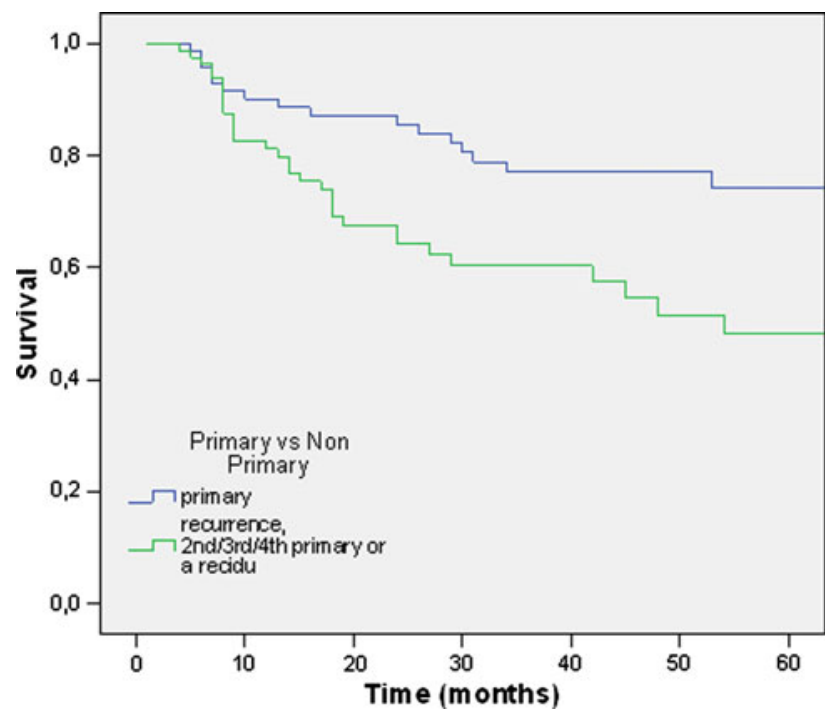

Fig. 3 Overall survival, comparing primary neoplasms versus nonprimary neoplasms

\section{Regional recurrence}

A total of 42 patients (24.7\%) had neck metastasis diagnosed by US and FNA and subsequently treated with neck dissection. Eighteen of these patients $(10.5 \%)$ had a neck metastasis combined with a second primary neoplasm or local recurrence. The staging of the neck after neck dissection was $\mathrm{N} 1$ in 26 patients, $\mathrm{N} 2 \mathrm{~b}$ in 14 patients, and N2c in 2 patients. There was no N3 or inoperable neck metastasis detected in this patient series.

\section{Adverse events}

Three patients had second degree burn wounds as a result of failure to comply by light protection guidelines. All of the burn wounds healed with conservative measurements. Nine patients had permanent discoloration at the injection site of mTHPC. Five patients had scar formation in the buccal mucosa leading to mild/moderate trismus. These patients were managed with conservative measures involving stretching exercises. Twelve patients had to receive temporary nasogastric sonda feeding for an average time of 5 days because of inability to swallow. The healing process after PDT takes 2-5 weeks and the wound heals with minimum scarring after this period. There were no systemic or serious adverse events.

\section{Discussion}

Photodynamic therapy has been used in head and neck oncology for some time with reported success [17-19, 2131]. Unfortunately, there are no studies comparing PDT with standard treatment methods, such as surgical resection and radiation therapy. Up to date, all data are over developing the technique of PDT and not actually comparing it with other methods. The overall clinical response rate after PDT as determined by the present analysis is below the reported success rates of surgery or radiation treatment [1-4]. The real reason is very difficult to determine. The patient groups are not comparable and furthermore additional treatments, such as re-excision for positive resection margins and postoperative radiation is often included in the disease-free survival analysis. The current analysis is focusing on finding favorable conditions for PDT and, therefore, excluding additional treatments, such as surgical resections from disease-free survival analysis (these patients are computed as failures). The ideal setup for such a comparison would be randomised study comparing surgical resection with PDT. However, for this purpose favorable situations, where PDT can be preferred over surgery, have to be identified.

The PDT literature fails to identify these favoured areas because of the small numbers of treated patients and the heterogeneity of treated groups making it impossible to give a clear answer to the question of what to treat and maybe more importantly what not to treat. These are all preliminary studies that were done to establish treatment protocols for PDT and subsequently treat patients to reach larger series. The results of such larger series are recently becoming available to us.

The longest experience is with photofrin-mediated PDT with the largest series published by Biel with 276 patients [28]. This series include laryngeal and oral cavity neoplasms with T1-3 N0 cancers. The CR rates are impressive with $91 \%$ for laryngeal neoplasms and $94 \%$ for oral cavity tumors. However, there is no subgroup analysis to show if certain subgroups of neoplasms react better. 
It is possible to extend the limits of PDT with mTHPC, which is activated with $652 \mathrm{~nm}$ light enabling deeper tissue penetration and providing treatment of deeper neoplasms [32]. A prospective multinational multi-institutional study with mTHPC-PDT was carried out with the participation of our institute [29]. The aimed group was patients with small neoplasms of the oral cavity with $<25 \mathrm{~mm}$ of diameter and $<5 \mathrm{~mm}$ depth of invasion. This group of well selected oral cavity tumors showed CR to MTHPC-PDT at a rate of $85 \%$ which is surprisingly lower than photofrin PDT [28].

The $5 \mathrm{~mm}$ cut-off limit is arbitrary. The international multicentre study carried out by D'Cruz et al. applied PDT to tumors deeper than $10 \mathrm{~mm}$ depth of invasion [30]. Treatment of deeper tumors (up to $50 \mathrm{~mm}$ ) was justified by the aim of the study, which was to provide palliation to incurable head and neck cancers by conventional methods. This great range of thickness enabled the authors to compare tumors with $<10 \mathrm{~mm}$ depth to tumors with $>10 \mathrm{~mm}$ depth. Among completely illuminated lesions with $<10 \mathrm{~mm}$ depth had a much better response than deeper tumors, with CR of 60 versus $26 \%$ and OR of 75 versus $40 \%$. It is a pity that they did not choose $5 \mathrm{~mm}$ depth as their comparison parameter, but we still get the idea that depth of the lesion is very important.

The choice method we use to determine the depth of the tumor is ultrasound. Endoscopic US is being used to stage several digestive system cancers, such as anal cancers with proven accuracy [33]. Shintani et al. [34] showed that intraoral US measurements of depth of tumor correlate well with histologic specimens. The whole surface of the tumor should be screened with US not to miss any spot deeper than $5 \mathrm{~mm}$. The inaccuracy of the US (if there is any) is compensated by PDT reaching $10 \mathrm{~mm}$ depth. The real problem is with oropharyngeal cancers, where the US probe cannot reach or cannot scan the entire surface. MRI can be used to determine depth. If the tumor cannot be seen on MR images or appear just as a contrast uptake, although there is no evidence to support it and it can be assumed that the tumor is $<5 \mathrm{~mm}$ deep. This method is by no means accurate and has to be replaced by another method. Introduction of newer methods, such as optical coherence tomography (OCT) might help in the future.

\section{Location of the neoplasm}

Oral cavity and oropharynx are not homogenous structures that have constant tissue characteristics. The tissue composition of the alveolar process is clearly different than that of the tongue or soft palate. Furthermore, some areas in the oral cavity and pharynx are flat while others have a complex geography. Multiple spots might have to be used to illuminate all the extensions of the neoplasm, theoretically making the chance of geographic miss greater.
It is, therefore, important to designate the favorable subsites of the oral cavity and oropharynx. The only sub-site that reacts significantly better is oral tongue. The reason is probably the relative homogeneity of the tissue with the absence of nearby bony structures, as well as the relative ease of delivering the light in a homogeneous manner on the flat surface.

Floor of mouth and soft palate have a more complex anatomy than the oral tongue with proximal bony structures (i.e. mandible and hard palate) probably causing lower CR rates. Buccal mucosa has a relatively flat surface providing ease of illumination with a rather homogenous structure with no apparent reason for a poorer outcome than the oral tongue. Although a statistic comparison is not carried out, buccal mucosa is also observed to have more scar tissue causing mild trismus (5/23 patients).

As can be expected, the neoplasms of the alveolar process have less CR rate than the overall mean. Alveolar process has a more complex anatomy with underlying mandible and has three surfaces making homogenous illumination harder to achieve. However, it can be argued that the OR rate of $73.7 \%$ helps in reducing the size of the neoplasm and enable a smaller excision subsequently.

The study by Hopper et al. [29] is the only publication reporting success rates for subsites, with CR rates of $89 \%$ for floor of mouth, $83 \%$ for lip, $93 \%$ for anterior tongue and $83 \%$ for buccal mucosa. There are publications that report failures in certain sites, but the numbers are very low to draw any conclusions [27].

The numbers of retromolar trigon, hard palate and nasal cavity neoplasms treated with PDT are not enough to do a statistical analysis $(9,8$ and 8 patients, respectively). However, the CR rates of the retromolar triangle $(66.7 \%)$ and hard palate $(62.5 \%)$ are near the overall mean $(70.8 \%)$.

The tumors of the oral tongue, which has the best results to PDT, are also usually easily resectable with minimum morbidity making PDT an unlikely candidate for initial therapy. Sites that would have functional problems after surgical resection, such as alveolar process and soft palate, although have lower response rates to PDT might be treated with PDT as initial treatment to avoid the functional problems, reserving surgery and/or radiation treatment for failures.

\section{T stage}

It can be an expected result because as the neoplasm area gets larger delivering light evenly gets harder and the risk for geographic miss gets greater. In our series, we do not observe such a difference. Although T1 tumors have a better CR rate than $\mathrm{T} 2$ tumors this is not statistically different (Table 1). Therefore, we can say that the size of the tumor does not make a difference as long as all of the tumor can be fully illuminated and the depth of invasion is $<5 \mathrm{~mm}$. 
In our series, Tis show a higher CR rate than $\mathrm{T} 1$ and $\mathrm{T} 2$ tumors with $79.5 \%$. Tis recur much earlier than $\mathrm{T} 1$ and $\mathrm{T} 2$ tumors (mean disease-free interval of 65.7 months for dysplasia versus 109.1 months for T1 and 113.4 months for T2 tumors) (Fig. 1). It is well known that patients with Tis of the upper aerodigestive tract are prone to develop new leukoplakia a number of times [35]. It should be kept in mind that PDT can be repeated a number of times with minimal morbidity to treat leukoplakia as they recur. The success of repeated treatments as lesions recur becomes evident by the similar survival rates of patients with Tis and T1 neoplasms. The difference in disease-free interval is made up for either by successful re-treatments or the relatively less lethal nature of Tis.

\section{Recurrences and multiple primary neoplasms}

Recurrences and multiple primary neoplasms pose a challenge to the head and neck oncologist. Most of these lesions occur in the previously irradiated or operated fields [36, 37]. The study by D'Cruz et al. focused on such patients who had refractory neoplasms of the head and neck area that were unsuccessfully treated or unsuitable for conventional treatments [30]. They report 38\% OR and 16\% CR. It should be noted that this study was a multicentre, multinational study with vague inclusion criteria, resulting in treatment of neoplasms that may not be suitable for surface PDT. When the lesions that were not fully illuminated and deeper than $10 \mathrm{~mm}$ were excluded they report an OR rate of $54 \%$ with $30 \%$ CR rate.

In our series, a total of 131 recurrent, second, or multiple primary neoplasms were treated with PDT. Even though non-primary neoplasms respond less favorably to PDT than primary neoplasms (65.9 vs. $77.9 \%$ CR, respectively, $P<0.05), 65.9 \% \mathrm{CR}$ is a considerable success if we take into account that the treated area received radiation in $48.1 \%$, chemoradiation in $22.7 \%$ and previous surgery in $75.6 \%$ of the patients. Furthermore, there is an $86.3 \%$ OR which means that an additional group of lesions decrease in size which makes subsequent surgical resection smaller. The difference with the study by D'Cruz et al. can be accounted to the more conservative selection criteria by our group.

\section{Management of neck nodes}

Elective treatment of N0 neck in case of early oral cavity cancers is a point of debate. Studies comparing elective neck dissection with wait and see policy show no difference in overall survival [38]. In experienced hands, US of the neck combined with FNA can reach a sensitivity of $73 \%$ and a specificity of $100 \%$ [39]. We adopted the wait and see policy for patients staged as N0 by US of the neck. Neck recurrences can be detected by regular US and FNA controls and the patients can receive subsequent neck dissections. This approach resulted in $24.7 \%$ patients receiving neck dissections. All of these patients had resectable neck nodes which were adequately removed after neck dissections. The number is quiet high if you take into consideration that the tumors in question are thinner than $5 \mathrm{~mm}$. This phenomenon can be explained with the association of the neck recurrence with second primary or recurrent tumors in almost half of the cases. It can be speculated that only $13 \%$ of the patients had neck recurrences that are associated with the tumor treated by PDT. Depending on the preference and policies of the center, elective neck dissection can be combined with PDT. The neck dissection has to be done either before PDT or 2-3 weeks after PDT. There is no evidence to prefer one timing over the other.

\section{Conclusion}

Our institutional experience supports the value of temoporfin-PDT in carefully selected patients. The success rate of PDT is independent of T stage as long as the depth of invasion does not exceed $5 \mathrm{~mm}$. Although tongue tumors respond best to PDT, areas that would have functional problems after resection such as palatum molle, alveolar process, retromolar trigon can be first treated with PDT to avoid morbidity and reserving surgery and/or radiation for failures/recurrences. Difficult to treat lesions such as recurrent neoplasms and multiple primary neoplasms located in previously irradiated or operated fields have a very acceptable $\mathrm{CR}$ rate with minimum morbidity, provided that they are carefully selected for eligibility.

Acknowledgments The study is not financially supported by any grant. The authors have no financial disclosures.

\section{Conflict of interest None.}

Open Access This article is distributed under the terms of the Creative Commons Attribution Noncommercial License which permits any noncommercial use, distribution, and reproduction in any medium, provided the original author(s) and source are credited.

\section{References}

1. Oliver RJ, Clarkson JE, Conway DI, Glenny A, Macluskey M, Pavitt $S$ et al (2007) Interventions for the treatment of oral and oropharyngeal cancers: surgical treatment. Cochrane Database Syst Rev 4:CD006205

2. Wildt J, Bjerrum P, Elbrond O (1989) Squamous cell carcinoma of the oral cavity: a retrospective analysis of treatment and prognosis. Clin Otolaryngol Allied Sci 14(2):107-113

3. Inagi $\mathrm{K}$, Takahashi H, Okamoto M, Nakayama M, Makoshi T, Nagai $\mathrm{H}$ (2002) Treatment effects in patients with squamous cell carcinoma of the oral cavity. Acta Otolaryngol Suppl 547:25-29 
4. Wolfensberger M, Zbaeren P, Dulguerov P, Muller W, Arnoux A, Schmid S (2001) Surgical treatment of early oral carcinoma-results of a prospective controlled multicenter study. Head Neck 23(7):525-530

5. Blair EA, Callender DL (1994) Head and neck cancer. The problem. Clin Plast Surg 21(1):1-7

6. Mlynarek AM, Rieger JM, Harris JR, O'Connell DA, Al Qahtani KH, Ansari K et al (2008) Methods of functional outcomes assessment following treatment of oral and oropharyngeal cancer: review of the literature. J Otolaryngol Head Neck Surg 37(1):2-10

7. Patton DW, Ali A, Davies R, Fardy MJ (1994) Oral rehabilitation and quality of life following the treatment of oral cancer. Dent Update 21(6):231-234

8. Rogers SN, Lowe D, Fisher SE, Brown JS, Vaughan ED (2002) Health-related quality of life and clinical function after primary surgery for oral cancer. Br J Oral Maxillofac Surg 40(1):11-18

9. Lee NY, Le QT (2008) New developments in radiation therapy for head and neck cancer: intensity-modulated radiation therapy and hypoxia targeting. Semin Oncol 35(3):236-250

10. Genden EM, Rinaldo A, Suarez C, Wei WI, Bradley PJ, Ferlito A (2004) Complications of free flap transfers for head and neck reconstruction following cancer resection. Oral Oncol 40(10):979-984

11. Langius A, Bjorvell H, Lind MG (1994) Functional status and coping in patients with oral and pharyngeal cancer before and after surgery. Head Neck 16(6):559-568

12. Glanzmann C, Gratz KW (1995) Radionecrosis of the mandibula: a retrospective analysis of the incidence and risk factors. Radiother Oncol 36(2):94-100

13. Yow CM, Chen JY, Mak NK, Cheung NH, Leung AW (2000) Cellular uptake, subcellular localization and photodamaging effect of temoporfin (mTHPC) in nasopharyngeal carcinoma cells: comparison with hematoporphyrin derivative. Cancer Lett 157(2):123131

14. Henderson BW, Bellnier DA (1989) Tissue localization of photosensitizers and the mechanism of photodynamic tissue destruction. Ciba Found Symp 146:112-125

15. Dougherty TJ, Marcus SL (1992) Photodynamic therapy. Eur J Cancer 28A(10):1734-1742

16. Hornung R, Walt H, Crompton NE, Keefe KA, Jentsch B, Perewusnyk $\mathrm{G}$ et al (1998) m-THPC-mediated photodynamic therapy (PDT) does not induce resistance to chemotherapy, radiotherapy or PDT on human breast cancer cells in vitro. Photochem Photobiol 68(4):569-574

17. Hopper C (2000) Photodynamic therapy: a clinical reality in the treatment of cancer. Lancet Oncol 1:212-219

18. Nyst HJ, Tan IB, Stewart FA, Balm AJ (2009) Is photodynamic therapy a good alternative to surgery and radiotherapy in the treatment of head and neck cancer? Photodiagn Photodyn Ther 6(1):3-11

19. Lou PJ, Jones L, Hopper C (2003) Clinical outcomes of photodynamic therapy for head-and-neck cancer. Technol Cancer Res Treat 2(4):311-317

20. Miller AB, Hoogstraten B, Staquet M, Winkler A (1981) Reporting results of cancer treatment. Cancer 47(1):207-214

21. Dilkes M, DeJode M, Rowntree-Taylor A, McGilligan J, Kenyon G, McKelvie P (1996) m-THPC photodynamic therapy for head and neck cancer. Lasers Med Sci 11(1):23-29

22. Kubler AC, Scheer M, Zoller JE (2001) Photodynamic therapy of head and neck cancer. Onkologie 24(3):230-237
23. Dilkes MG, Benjamin E, Ovaisi S, Banerjee AS (2003) Treatment of primary mucosal head and neck squamous cell carcinoma using photodynamic therapy: results after 25 treated cases. J Laryngol Otol 117(9):713-717

24. Fan KF, Hopper C, Speight PM, Buonaccorsi GA, Bown SG (1997) Photodynamic therapy using mTHPC for malignant disease in the oral cavity. Int J Cancer 73(1):25-32

25. Lorenz KJ, Maier H (2009) Photodynamic therapy with meta-tetrahydroxyphenylchlorin $(\operatorname{Foscan}((\mathrm{R})))$ in the management of squamous cell carcinoma of the head and neck: experience with 35 patients. Eur Arch Otorhinolaryngol

26. Wenig BL, Kurtzman DM, Grossweiner LI, Mafee MF, Harris DM, Lobraico RV et al (1990) Photodynamic therapy in the treatment of squamous cell carcinoma of the head and neck. Arch Otolaryngol Head Neck Surg 116(11):1267-1270

27. Kulapaditharom B, Boonkitticharoen V (2000) Photodynamic therapy in management of head and neck cancers and precancerous lesions. J Med Assoc Thai 83(3):249-258

28. Biel MA (2007) Photodynamic therapy treatment of early oral and laryngeal cancers. Photochem Photobiol 83(5):1063-1068

29. Hopper C, Kubler A, Lewis H, Tan IB, Putnam G (2004) mTHPCmediated photodynamic therapy for early oral squamous cell carcinoma. Int J Cancer 111(1):138-146

30. D'Cruz AK, Robinson MH, Biel MA (2004) mTHPC-mediated photodynamic therapy in patients with advanced, incurable head and neck cancer: a multicenter study of 128 patients. Head Neck 26(3):232-240

31. Copper MP, Tan IB, Oppelaar H, Ruevekamp MC, Stewart FA (2003) Meta-tetra(hydroxyhenyl)chlorin photodynamic therapy in early-stage squamous cell carcinoma of the head and neck. Arch Otolaryngol Head Neck Surg 129(7):709-711

32. Berenbaum MC, Akande SL, Bonnett R, Kaur H, Ioannou S, White RD et al (1986) meso-Tetra(hydroxyphenyl)porphyrins, a new class of potent tumour photosensitisers with favourable selectivity. Br J Cancer 54(5):717-725

33. Schizas AM, Williams AB, Meenan J (2009) Endosonographic staging of lower intestinal malignancy. Best Pract Res Clin Gastroenterol 23(5):663-670 Review

34. Shintani S, Yoshihama Y, Ueyama Y, Terakado N, Kamei S, Fijimoto Y, Hasegawa Y, Matsuura H, Matsumura T (2001) The usefulness of intraoral ultrasonography in the evaluation of oral cancer. Int J Oral Maxillofac Surg 30(2):139-143

35. Braakhuis BJ, Tabor MP, Kummer JA, Leemans CR, Brakenhoff RH (2003) A genetic explanation of slaughter's concept of field cancerization: evidence and clinical implications. Cancer Res 63(8): 1727-1730

36. Ridge JA (1993) Squamous cancer of the head and neck: surgical treatment of local and regional recurrence. Semin Oncol 20(5):419-429

37. Leon X, Quer M, Diez S, Orus C, Lopez-Pousa A, Burgues J (1999) Second neoplasm in patients with head and neck cancer. Head Neck 21(3):204-210

38. Nieuwenhuis EJ, Castelijns JA, Pijpers R, van den Brekel MW, Brakenhoff RH, van der Waal I, Snow GB, Leemans CR (2002) Wait-and-see policy for the N0 neck in early-stage oral and oropharyngeal squamous cell carcinoma using ultrasonography-guided cytology: is there a role for identification of the sentinel node? Head Neck 24(3):282-289

39. van den Brekel MW, Castelijns JA, Stel HV et al (1991) Occult metastatic neck disease: detection with US and US-guided fine needle aspiration cytology. Radiology 180:457-461 